CALT-68-2169

hep-th/9804067

April 1998

\title{
M-momentum Transfer between Gravitons, Membranes, and Fivebranes as Perturbative Gauge Theory Processes
}

\author{
Esko Keski-Vakkuri ${ }^{1}$ and Per Kraus ${ }^{2}$ \\ California Institute of Technology \\ Pasadena CA 91125, USA \\ email: esko or perkraus@theory.caltech.edu
}

\begin{abstract}
:
Polchinski and Pouliot have shown that M-momentum transfer between membranes in supergravity can be understood as a non-perturbative instanton effect in gauge theory. Here we consider a dual process: electric flux transmission between D-branes. We show that this process can be described in perturbation theory as virtual string pair creation, and is closely related to Schwinger's treatment of the pair creation of charged particles in a uniform electric field. Through the application of dualities, our perturbative calculation gives results for various nonperturbative amplitudes, including M-momentum transfer between gravitons, membranes and longitudinal fivebranes. Thus perturbation theory plus dualities are sufficient to demonstrate agreement between supergravity and gauge theory for a number of M-momentum transferring processes. A variety of other processes where branes are transmitted between branes, e.g. $(p, q)$ string transmission in IIB theory, can also be studied. We discuss the implications of our results for proving the 11 dimensional Lorentz invariance of Matrix theory.
\end{abstract}

${ }^{1}$ Work supported in part by a DOE grant DE-FG03-92-ER40701.

${ }^{2}$ Work supported in part by a DOE grant DE-FG03-92-ER40701 and by the DuBridge Foundation. 


\section{Introduction}

In the flat, eleven dimensional limit of M-theory, scattering amplitudes involving the exchange of momentum in the eleventh direction are simply related by rotational invariance to processes involving purely transverse momentum exchange. However, in the gauge theory description of M-theory used in Matrix Theory [1], this rotational symmetry is made highly non-manifest and must arise from relatively complicated non-perturbative effects, if it is there at all. Thus while numerous checks have been made of the correspondence between transverse momentum exchange and perturbative gauge theory processes, so far the successful description of M-momentum transfer has been limited to a single case involving the scattering of membranes [2, 3]. In this example, it was shown by Polchinski and Pouliot that including instanton effects in the relevant $2+1$ super Yang-Mills theory円 rendered the eleventh dimension on an equal footing with the other ten. A T-dual version of this process was considered by Banks et. al. [5], who showed that by wrapping the membranes on a torus which shrinks to zero size one obtains Y-momentum transfer between gravitons in IIB theory. Unfortunately, it is a difficult and unsolved problem to carry out the analogous calculations for cases other than membranes.

On the other hand, since the relation between the transverse and eleventh dimensions in M-theory maps on to the relation between perturbative and non-perturbative effects in gauge theory, we might hope to make further progress by application of duality transformations. Many non-perturbative results in the gauge theory can be obtained by starting from a perturbative result and applying duality. In the present work we exploit this approach in order to 1) rederive the result of Polchinski and Pouliot from a purely perturbative computation 2) show how various other non-perturbative amplitudes can be similarly computed, including other M-momentum transferring processes and 3) clarify the problem of exhibiting the full eleven dimensional Lorentz invariance from the gauge theory description. Something of a similar approach has been discussed in the context of describing transverse 5-branes in Matrix Theory [6, 7].

The recovery of the Polchinski-Pouliot amplitude from a perturbative calculation proceeds as follows. We first note that in ten dimensions the process corresponds to the exchange of D0-branes between D2-branes. By compactifying a transverse direction and performing T-duality, we obtain D1-brane exchange between D3-branes. Finally, S-duality maps the configuration to the exchange of fundamental strings between D3-branes, a pro-

\footnotetext{
${ }^{1}$ For additional discussion of the action used in [2], see [4] and De Boer et. al. in [3].
} 
cess which has a purely perturbative gauge theory description. In section 3, we perform the perturbative gauge theory calculation, which is closely related to Schwinger's calculation of the pair production of charged particles in an electric field [ $[\mathbb{B}]$, implement the duality transformations, and show in section 4 that the result indeed agrees with the Polchinski-Pouliot amplitude.

Fundamental string exchange between D3-branes is easily generalized to the case of other Dp-branes. Applying duality transformations then allows one to generate amplitudes for a wide variety of non-perturbative processes, as we discuss in section 5 . This includes, for instance, M-momentum exchange between D0-branes or between D4-branes. For such amplitudes, there has been little progress based on the approach of directly evaluating the relevant non-perturbative effects. For instance, the calculation in the case of D0-branes seems to require detailed knowledge of the ill-understood bound state wavefunction describing a cluster of D0-branes. However, by using dualities to relate the amplitudes to other perturbative effects, some progress can be made.

Therefore we see that there is no impediment to computing M-momentum transfer in a number of different cases. What does this say about the prospects for demonstrating eleven dimensional Lorentz invariance in general? Certainly, a proof of Lorentz invariance should include supplying a means of computing an arbitrary M-momentum transferring amplitude. For our purposes, it is essential to differentiate between two types of such processes: those to which duality can be applied to yield a perturbative amplitude, and those which cannot be so transformed. The results of [2] and the present work are evidence that Lorentz invariance is recovered for the first type of processes, but there is presently no evidence regarding the second type.

The processes considered here - and every successfully treated example existing in the literature - are such that the objects exchanged in a given scattering are of a single type. This is sufficient to guarantee that the calculations can be reduced to perturbation theory with the help of dualities. This is no longer true when one consider the exchange of multiple kinds of objects in a given amplitude. For example, in the case of D3-brane scattering we can compute amplitudes corresponding to the exchange of F-strings or of D-strings; however, we cannot use our techniques to compute the second type of amplitude which involves the exchange of both F and D strings simultaneously. Specifically, the F-string case follows from a perturbative computation, and the D-string case is then obtained by applying a duality transformation, but an amplitude involving the exchange 
of two strings, one $\mathrm{F}$ and one D, I cannot be transformed into a perturbative process. To demonstrate that the latter type of amplitudes are consistent with eleven dimensional Lorentz invariance one is forced to do a non-perturbative gauge theory calculation. Without explicitly performing such a calculation, it is not possible to use dualities to argue for Lorentz invariance in the general case. As another example, one can consider the scattering of three membranes with different amounts of M-momentum transferred between each pair. Duality transformations could be used to map a given subprocess to a perturbative process, but one would still require a non-perturbative calculation to correctly evaluate the total amplitude.

The fact that perturbative results plus dualities are enough to establish Lorentz invariance for a variety of amplitudes is an encouraging sign that the symmetry exists in the gauge theory. In our opinion, though, a true test of this conjecture will involve evaluating an amplitude which cannot be made perturbative through any sequence of dualities. Only then will we have a result which is not directly implied by perturbation theory plus a symmetry.

\section{M-momentum transfer in supergravity}

We begin by writing down the type of supergravity amplitude which we wish to recover from gauge theory. For definiteness, we consider the case of M-momentum transfer between membranes. The idea is to calculate, at the level of classical supergravity, the contribution to the potential between two membranes due to the exchange of momentum in the eleventh direction. The calculation is a slight modification of the one appearing in [2] - we take the membranes to have a nonzero relative velocity in the eleventh direction and zero relative velocity in the transverse directions, whereas in [2] the opposite configuration is considered. To perform the calculation we use the well known source-probe method: we first write down the field configuration produced by one of the membranes (the source) and then introduce the second membrane by coupling it to the background according to the supermembrane action. We then compactify the eleventh direction and a transverse direction by replacing the single source membrane by a periodic array of membranes in the compact directions. Finally, we perform a Poisson resummation to exhibit the sum over exchanged states in a form which is conveniently compared with

\footnotetext{
${ }^{2}$ Here we do not mean a $(1,1)$ string, which we are able to treat with our methods, but rather two strings: a $(1,0)$ and a $(0,1)$.
} 
gauge theory results.

The metric produced by a membrane in non-compactified eleven dimensional space 1 is 9

$$
\begin{gathered}
d s^{2}=H_{2}^{-2 / 3}\left[d t^{2}-\left(d x^{1}\right)^{2}-\left(d x^{2}\right)^{2}\right]-H_{2}^{1 / 3}\left[\left(d x^{3}\right)^{2}+\cdots+\left(d x^{11}\right)^{2}\right] . \\
H_{2}=1+\frac{Q_{2}^{(11)}}{r^{6}} \quad ; \quad r^{2}=\left(x^{3}\right)^{2}+\cdots+\left(x^{11}\right)^{2} .
\end{gathered}
$$

The action of the probe membrane is given by

$$
S_{2}=-T_{2}^{(11)} \int d^{3} x\left\{\sqrt{\operatorname{det}\left(g_{M N} \partial_{\mu} X^{M} \partial_{\nu} X^{N}\right)}-H^{(3)}\right\},
$$

where the determinant acts on the worldvolume indices $\mu, \nu$. The tension and charge of the membrane are related to the Planck mass by

$$
T_{2}^{(11)} Q_{2}^{(11)}=\frac{8}{M_{11}^{3}}
$$

We take the probe to lie in the $x^{0}, x^{1}, x^{2}$ plane, and to be moving with constant velocity $v$ in the eleventh direction. Evaluating the action in static gauge, the leading interaction contribution comes from the $v^{4}$ term and reads

$$
S_{2}=\frac{1}{M_{11}^{3}} \int d^{3} x \frac{v^{4}}{r^{6}}
$$

To compactify $x^{3}, x^{11}$, we replace the single source membrane by a periodic array:

$$
\frac{1}{r^{6}} \rightarrow \sum_{n_{3}, n_{11}} \frac{1}{\left[r^{2}+\left(2 \pi n_{3} R_{3}\right)^{2}+\left(2 \pi n_{11} R_{11}\right)^{2}\right]^{3}},
$$

where on the right hand side $r^{2}=\left(x^{4}\right)^{2}+\cdots+\left(x^{9}\right)^{2}$. Since we are not presently interested in the exchange of momentum in the $x^{3}$ direction, we will take the limit of large $R_{3}$ and so replace the sum over $n_{3}$ by an integral. In addition, we use the Poisson resummation formula to rewrite the sum over $n_{11}$. We then have,

$$
\frac{1}{r^{6}} \rightarrow \frac{3}{16 R_{3}} \frac{1}{2 \pi R_{11}} \sum_{m} \int_{-\infty}^{\infty} d x \frac{e^{i m x / R_{11}}}{\left(r^{2}+x^{2}\right)^{5 / 2}}=\frac{1}{16 \pi} \frac{1}{R_{3} R_{11}^{3}} \frac{1}{r^{2}} \sum_{m} m^{2} K_{2}\left(|m| r / R_{11}\right),
$$

where $K_{2}$ is a modified Bessel function. For the action we then find

$$
S_{\mathrm{eff}}=\int d^{3} x \frac{1}{16 \pi} \frac{1}{M_{11}^{3} R_{3} R_{11}^{3}} \frac{v^{4}}{r^{2}} \sum_{m} m^{2} K_{2}\left(|m| r / R_{11}\right) .
$$

\footnotetext{
${ }^{3}$ We take the coordinates to be $t, x^{1}, \ldots, x^{9}, x^{11}$
} 
The $m$ 'th term in the sum corresponds to the contribution from the exchange of $m$ units of M-momentum, This is seen from the asymptotic form for large argument of the modified Bessel function

$$
K_{2}\left(|m| r / R_{11}\right) \sim \sqrt{\frac{\pi R_{11}}{2|m| r}} e^{-|m| r / R_{11}},
$$

so that the rate of fall off matches with the propagator of a massive Kaluza-Klein state. Keeping only the leading $m=1 \mathrm{term}$, the result for large transverse separation is

$$
S_{\text {eff }}=\int d^{3} x \frac{1}{16 \sqrt{2 \pi}} \frac{1}{M_{11}^{3} R_{3} R_{11}^{5 / 2}} \frac{v^{4}}{r^{5 / 2}} e^{-r / R_{11}}
$$

For comparison with gauge theory, it is helpful to rexpress the result in ten dimensional string units using

$$
M_{11}^{3}=\frac{1}{\left(\alpha^{\prime}\right)^{3 / 2} g_{s t}} \quad ; \quad R_{11}=\sqrt{\alpha^{\prime}} g_{s t} .
$$

In these units,

$$
S_{\text {eff }}=\int d^{3} x \frac{1}{16 \sqrt{2 \pi}} \frac{\left(\alpha^{\prime}\right)^{1 / 4}}{g_{s t}^{3 / 2} R_{3}} \frac{v^{4}}{r^{5 / 2}} e^{-\frac{r}{\sqrt{\alpha^{\prime}} g_{s t}}}
$$

The appearance of $1 / g_{s t}$ in the exponential indicates a nonperturbative effect in the gauge theory description. In the case of nonzero transverse velocities, the above formula was reproduced from an instanton calculation in [2]. Our goal in the remainder of this work is to demonstrate how this and other amplitudes can be obtained starting from purely perturbative calculations, which are easier to perform.

\section{Gauge theory calculation}

In this section we calculate the contribution to the one-loop gauge theory effective action from the exchange of F-strings between bound states of Dp-branes and F-strings. The calculation is perturbative in gauge theory and in the string coupling $g_{s t}$, though the result will be seen to be non-perturbative in terms of $\alpha^{\prime}$. In the next section we will show that applying dualities to the result yields the supergravity amplitude (10).

The basic description of this process is as follows. Consider two $\mathrm{D} p$-branes, separated by a transverse distance $r$, and with $q_{1}, q_{2}$ F-strings bound to them. Let one of the $p$ spatial directions be compact with length $2 \pi R$, and let the F-strings wind around this direction. The F-strings are described in the gauge theory as electric flux in the unbroken $\mathrm{U}(1)$, with the electric field being proportional to the difference in F-string number, $q_{1}-q_{2}$. Massive 
excitations with $\mathrm{U}(1)$ charge in the gauge theory correspond to open strings stretching between the two D-branes. Thus there is an analogue of the Schwinger effect given by pair creation of open stringst. The creation of real strings gives an imaginary contribution to the effective action [11]. If the pair propagates only in imaginary time as virtual strings, the contribution to the effective action is real.

Since one of the spatial directions is periodic, the strings (which propagate in opposite directions) can propagate around the circle $m$ times before annihilating. Consider a process where they wind once around the circle:

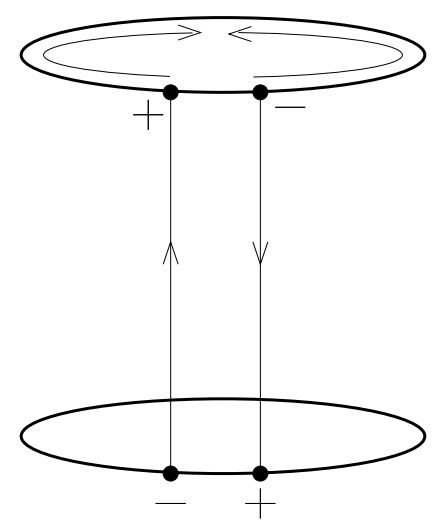

Figure : F-string transmission. A virtual string pair is created between the Dbranes, propagates around the compact direction and then disappears. The electric flux lines (not shown) running from positive charges to negative charges will remain after the pair has disappeared. Thus the electric flux increases (decreases) on the upper (lower) brane by one unit, corresponding to F-string transfer from the lower brane to the upper brane.

The electric field lines between the endpoints of the string close around the circle, so the electric flux changes by +1 unit on one D-brane and by -1 unit on the other D-brane. But since the electric flux counts the number of F-strings bound to the brane, this means that one F-string has transferred from one D-brane to the other in this process. Similarly, a path with winding number $m$ transfers $m$ F-strings from D-brane to D-brane.

We now move to the gauge theory calculation. For the most part, the calculation follows the well known method introduced in [11]. However, there will be a departure from the standard treatment halfway through when we take into account the F-string transmission.

\footnotetext{
${ }^{4}$ A pair creation effect can also occur on a single D-brane, if the electric field on it exceeds a critical value [10].
} 
We consider $p_{1}+p_{2} \mathrm{D} p$-branes in the $x^{1}, \ldots, x^{p}$ plane. We then compactify $x^{1}, \ldots x^{p}$ on a torus of radii $R_{1}, \ldots R_{p}$, and add $q_{1}+q_{2} \mathrm{~F}$-strings winding along the direction $x^{p}$. The world volume gauge theory is the $D=p+1$ dimensional $\mathrm{U}\left(p_{1}+p_{2}\right)$ Super-Yang-Mills-Higgs theory with the Lagrangian

$$
\mathcal{L}=\frac{1}{g_{Y M}^{2}} \operatorname{Tr}\left\{-\frac{1}{4} F^{\mu \nu} F_{\mu \nu}+\frac{1}{2} D^{\mu} \phi_{i} D_{\mu} \phi_{i}+\frac{1}{4}\left[\phi_{i}, \phi_{j}\right]^{2}+\text { fermions }\right\} .
$$

We separate the $\mathrm{D} p$-branes by a transverse distance $r$ in the $x^{D}$-direction into two groups with $p_{1}$ and $p_{2}$ branes by giving the Higgs field $\phi_{D}$ a vacuum expectation value

$$
\bar{\phi}_{D}=\left(\begin{array}{ll}
\frac{r}{2} \mathbf{1}_{p_{1}} & \\
& -\frac{r}{2} \mathbf{1}_{p_{2}}
\end{array}\right) .
$$

This breaks the $\mathrm{U}\left(p_{1}+p_{2}\right)$ symmetry to $\mathrm{U}\left(p_{1}\right) \times \mathrm{U}\left(p_{2}\right)$.

The $q_{1}, q_{2}$ F-strings bound to the $p_{1}, p_{2}$ D-branes are represented by two background electric fields

$$
E_{1}=q_{1} / R_{p} ; E_{2}=q_{2} / R_{p} .
$$

That is to say, there is a background gauge field

$$
\begin{aligned}
& \bar{A}_{\mu}=\mathbf{0}_{p_{1}+p_{2}}, \mu=0 \ldots p-1 ; \\
& \bar{A}_{p}=\left(\begin{array}{ll}
E_{1} t \mathbf{1}_{p_{1}} & \\
& E_{2} t \mathbf{1}_{p_{2}}
\end{array}\right) .
\end{aligned}
$$

Next, we find the effective potential between the D-brane bound states by integrating out the massive field fluctuations about the background (14),(16). We are interested only in contributions at the one loop level. Since refs. [12, 13] give the result for general D0-brane backgrounds, it is convenenient to T-dualize our configuration to that of D0-branes, in which case the gauge field components $A_{i}$ are converted to Higgs fields $\phi_{i}$ by the relation [14, 6]

$$
i \partial_{i}+A_{i} \leftrightarrow \phi_{i},
$$

The resulting effective action $S_{\text {eff }}$ takes the following functional determinant form:

$$
\begin{aligned}
e^{i S_{\text {eff }}=} & \operatorname{det}(H-i 2 E)^{-1} \operatorname{det}(H+i 2 E)^{-1} \operatorname{det}(H)^{-6} \\
& \operatorname{det}(H+i E)^{4} \operatorname{det}(H-i E)^{4}
\end{aligned}
$$

where the operator $H$ is

$$
H=\left[-\partial_{t}^{2}+\partial_{1}^{2}+\cdots+\partial_{p-1}^{2}+\left(\partial_{p}-i E t\right)^{2}+r^{2}\right] \cdot \mathbf{1}_{p_{1} p_{2}}
$$


and $E$ is the difference of the two electric fields $E_{1}, E_{2}$,

$$
E=\left[E_{1}-E_{2}\right] \cdot \mathbf{1}_{p_{1} p_{2}}
$$

As expected, only the difference of the electric fields appears. Note that in contrast to the usual practise in the literature, in the above we work in real time $t$. We now use Schwinger's trick to exponentiate the determinants:

$$
S_{\text {eff }}=i p_{1} p_{2} \int_{0}^{\infty} \frac{d s}{s}(6+2 \cosh 2 E s-8 \cosh E s) \operatorname{tr} e^{-i H s} .
$$

(Note that now $H, E$ no longer contain the unit matrix $\mathbf{1}_{p_{1} p_{2}}$ - it has been traced over to give the factor $p_{1} p_{2}$.)

To this point the calculation has been standard procedure; the new twist comes from evaluating $\operatorname{tre}^{-i H s}$. In the coordinate representation, the trace becomes an integral over a quantum mechanical propagator $K(x, y ; s)$ :

$$
\operatorname{tr} e^{-i H s}=\int d^{D} x\left\langle x\left|e^{-i H s}\right| x\right\rangle \equiv \int d^{D} x K(x, x ; s) .
$$

The operator $H$ is identical to the small fluctuation operator for a massive charged scalar field minimally coupled to a uniform background electric field $E$.

In noncompact space, the propagator $K_{H}(x, y ; s)$ for a charged particle in an electric field can be found in e.g. [15, 16],

$$
K_{H}(x, y ; s)=\frac{i E}{(4 \pi i)^{D / 2} s^{D / 2-1} \sinh (E s)} e^{-i r^{2} s+i(E / 4)\left[\operatorname{coth}(E s)\left(\left(y^{p}-x^{p}\right)^{2}-\left(y^{0}-x^{0}\right)^{2}\right)+2\left(x^{p} y^{0}-x^{0} y^{p}\right)\right]} .
$$

For a path corresponding to an instanton we take

$$
x^{0}=y^{0}=t .
$$

Since we take space to be a torus, the propagator $K(x, x ; s)$ in (21) is a sum of contributions with different winding number $m \in \mathbf{Z}$ corresponding to paths which wind $m$ times around the $x_{p}$-cycle:

$$
K(x, x ; s)=\sum_{m=-\infty}^{\infty} K_{H}\left(x, x+2 \pi m R_{p} e_{p} ; s\right)
$$

where $K_{H}$ is the propagator (22) with (23):

$$
K_{H}\left(x, x+2 \pi m R_{p} ; s\right)=\frac{i E}{(4 \pi i)^{D / 2} s^{D / 2-1} \sinh (E s)} e^{-i r^{2} s+i E m^{2} \pi^{2} R^{2} \operatorname{coth}(E s)-i E \pi m R t} .
$$


Then, after rescaling $s \rightarrow s / r^{2}$, the effective action reads as follows,

$$
\begin{array}{r}
S_{\text {eff }}=i p_{1} p_{2} \int d^{D} x \int_{0}^{\infty} d s \frac{i E\left(6+2 \cosh \left(2 E s / r^{2}\right)-8 \cosh \left(E s / r^{2}\right)\right)}{(4 \pi i s)^{D / 2} \sinh \left(E s / r^{2}\right)} \\
\sum_{m=-\infty}^{\infty} \exp \left\{-i s+i E m^{2} \pi^{2} R_{p}^{2} \operatorname{coth}\left(E s / r^{2}\right)-i E \pi m R_{p} t\right\} .
\end{array}
$$

Assuming that the bound states are well separated,

$$
\frac{E}{r^{2}} \ll 1
$$

so we can expand in $E / r^{2}$. we find the leading contribution to the effective action

$$
S_{\text {eff }}=\int d^{D} x \frac{\pi^{4-D} p_{1} p_{2} E^{4} R_{p}^{4-(D / 2)}}{2^{D-1} r^{4-(D / 2)}} \sum_{m=-\infty}^{\infty}|m|^{4-(D / 2)} K_{4-(D / 2)}\left(2 \pi|m| R_{p} r\right) e^{-i E \pi m R_{p} t}
$$

where $K_{4-(D / 2)}$ is a modified Bessel function, with asymptotic behavior for large argumentf

$$
K_{4-(D / 2)}\left(2 \pi|m| R_{p} r\right) \sim \frac{1}{2 \sqrt{|m| R_{p} r}} e^{-2 \pi|m| R_{p} r} .
$$

In the above, we have set $2 \pi \alpha^{\prime}=1$. In the next section, it will be useful to have the result for D3-branes $(D=4)$, keeping track of correct dimensions. After restoring $\alpha^{\prime}$, and setting $p_{i}=m=1, t=0$ for simplicity, the asymptotic form of the effective action becomes

$$
S_{\text {eff }}=\int \frac{d^{4} x}{\left(2 \pi \alpha^{\prime}\right)^{2}} \frac{\left(2 \pi \alpha^{\prime} E\right)^{4} R_{3}^{3 / 2} \sqrt{2 \pi \alpha^{\prime}}}{16 r^{5 / 2}} e^{-R_{3} r / \alpha^{\prime}} .
$$

The appearance of $1 / \alpha^{\prime}$ in the exponent indicates that the result is non-perturbative in terms of $\alpha^{\prime}$.

\section{M-momentum transfer as F-string transfer}

We now show how to recover the non-perturbative result for M-momentum exchange between membranes starting from the perturbative process of fundamental string exchange between D3-branes. We begin with two D3-branes in the $x^{1}, x^{2}, x^{3}$ plane, where $x^{3}$ is compactified on a circle of radius $R_{3}$. The D3-branes have worldvolume electric fields

\footnotetext{
${ }^{5}$ One can also check that shrinking a compact transverse direction to zero changes the power law falloff of (27) correctly for the lower dimension. For zero-brane scattering, this was discussed in [17].
} 
pointing in the $x^{3}$ direction; we denote $E=E_{1}-E_{2}$ as the difference in the two electric fields. The effective potential due to the exchange of F-strings wrapped around the $x^{3}$ direction is given by (28). We will perform the following duality transformations:

1) S-duality. Process then corresponds to exchange of D-strings wrapped on on $x^{3}$ between D3-branes with relative magnetic fields $B=E$.

2) T-duality in $x^{3}$ direction. Process then corresponds to exchange of D0-branes between D2-branes with relative magnetic fields $B$.

The final process is precisely M-momentum transfer between membranes, and is a non-perturbative effect arising from instantons.

The first step, S-duality, takes $g_{s t} \rightarrow 1 / g_{s t}, E \rightarrow B$, and rescales lengths as measured in the string metric: $x \rightarrow x / \sqrt{g_{s t}}$. We will express results in terms of the radius of the eleventh dimension, $R_{11}$, using (11). We obtain for the action

$$
S_{\text {eff }}=\int d^{4} x \frac{\left(\alpha^{\prime}\right)^{3 / 4}}{16\left(2 \pi \alpha^{\prime}\right)^{3 / 2}} \frac{R_{3}^{3 / 2}}{R_{11}^{3 / 2}} \frac{\left(2 \pi \alpha^{\prime} B\right)^{4}}{r^{5 / 2}} e^{-\frac{R_{3} r}{\sqrt{\alpha^{\prime}} R_{11}}}
$$

The second step, T-duality in direction $x^{3}$, takes

$$
R_{3} \rightarrow \frac{\alpha^{\prime}}{R_{3}} \quad ; \quad R_{11} \rightarrow \frac{\sqrt{\alpha^{\prime}}}{R_{3}} R_{11}
$$

The action now becomes

$$
S_{\text {eff }}=\int d^{3} x \frac{\left(\alpha^{\prime}\right)^{3 / 2}}{16 \sqrt{2 \pi \alpha^{\prime}}} \frac{1}{R_{11}^{3 / 2} R_{3}} \frac{\left(2 \pi \alpha^{\prime} B\right)^{4}}{r^{5 / 2}} e^{-\frac{r}{R_{11}}}
$$

where the integral over the $x^{3}$ direction has been performed to give a factor of $2 \pi\left(\alpha^{\prime} / R_{3}\right)$.

Finally, we rexpress the result in terms of the eleven dimensional Planck mass using (11), and reinterpret the magnetic field as velocity in the eleventh direction

$$
2 \pi \alpha^{\prime} B \rightarrow v
$$

Our final result for the effective action is then

$$
S_{\text {eff }}=\int d^{3} x \frac{1}{16 \sqrt{2 \pi}} \frac{1}{M_{11}^{3} R_{3} R_{11}^{5 / 2}} \frac{v^{4}}{r^{5 / 2}} e^{-\frac{r}{R_{11}}} .
$$

we see that the result agrees with (10). 


\section{Other transfer processes}

After having shown that nonperturbative M-momentum transfer between D2-branes can be related to the perturbative F-string transmission process, a natural question to ask is what other processes can be understood by relating them to the F-string transmission by various dualities. In this section we discuss some other examples.

Let us take as a starting point the case $p=1$ of section 2: transfer of $\mathrm{m}$ F-strings between $(p, q)$-strings in IIB theory. Recall that $(p, q)$-strings are bound states of $p$ Dstrings and $q$ F-strings and that they are stable against decay into multiple strings if $p$ and $q$ are relatively prime: $\operatorname{gcd}(p, q)=1$ [18]. Then, note that any such $(p, q)$-string can always be mapped to a fundamental string by an $\mathrm{SL}(2, \mathrm{Z})$ transformation:

$$
\left(\begin{array}{cc}
q & -p \\
a & b
\end{array}\right)\left(\begin{array}{l}
p \\
q
\end{array}\right)=\left(\begin{array}{l}
0 \\
1
\end{array}\right) \text {. }
$$

The integers $a, b$ such that

$$
a p+b q=1
$$

can be found using Euclid's algorithm. Obviously, $m$ (p,q)-strings map to $m$ F-strings. Therefore, a $(p, q)$-string transmission process

$$
\left(p_{1}, q_{1}\right) \stackrel{(p, q)}{\longrightarrow}\left(p_{2}, q_{2}\right)
$$

(meaning $(p, q)$-strings transferred between a $\left(p_{1}, q_{1}\right)$-string and a $\left(p_{2}, q_{2}\right)$-string) is related to the simple F-string transmission process of section 2 by a transformation (34). [

Suppose that the $(p, q)$-strings wind around compact direction $x_{1}$. T-duality along this direction then maps the process (36) to one in which D0-branes are transferred between clusters of D0-branes:

$$
p_{1} D 0+v_{1} \stackrel{p D 0+v}{\longrightarrow} p_{2} D 0+v_{2},
$$

with transverse velocities $v_{i}=q_{i} / R_{1}$ and $v=q / R_{1}$ where $R_{1}$ is the radius of the compact direction $x_{1}$. In other words, we can calculate M-momentum transfer between gravitons. Such a calculation seems to be very difficult to perform directly without a detailed understanding of the properties of marginally bound states of D0-branes.

\footnotetext{
${ }^{6}$ Some restrictions apply here: we assume that $q p_{i}-p q_{i}>0$ so that after the $\mathrm{SL}(2, \mathrm{Z})$ transformation (34) the images of the bound states $\left(p_{i}, q_{i}\right)$ have a strictly positive D-string charge. Then we can avoid dealing with pairs of D-strings and D-antistrings.

${ }^{7} \mathrm{~A}$ interesting case to consider is when the final string has $\left(p_{2}, q_{2}\right)$ not relatively prime. In this case one could end up with either with a marginally bound $\left(p_{2}, q_{2}\right)$ string, or a multiple string state corresponding to its constituents. How to distinguish such final states in our formalism is not clear.
} 
Now suppose that the directions $x_{2}, x_{3}$ are also compact. Then, T-duality in directions $x_{2}, x_{3}\left(T_{2} T_{3}\right.$ for short) relates the process (36) to

$$
p_{1} D 3+q_{1} F 1 \stackrel{p D 3+q F 1}{\longrightarrow} \stackrel{\longrightarrow}{\longrightarrow} p_{2} D 3+q_{2} F 1 .
$$

(38) is the generalized version of the F-string transfer of section 2: the bound states can exchange D3-branes as well as F-strings.

Continuing with S-duality and T-duality $T_{1}$, (38) in turn maps to a more general version of the M-momentum transfer process of [2]:

$$
p_{1} D 2+q_{1} D 0 \stackrel{p D 2+q D 0}{\longrightarrow} \underset{\longrightarrow}{\longrightarrow} p_{2} D 2+q_{2} D 0
$$

where both D2-branes and D0-branes can be transferred.

Another T-duality $T_{2}$ maps (39) to a process involving diagonal D-strings winding $p, q$ times along the directions $x_{3}, x_{2}$. We use the homology notation $p(3)+q(2)$ for such diagonal strings [19]. We obtain

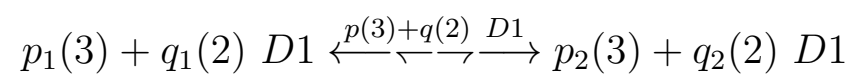

where the diagonal D1-strings change their winding.

Now, if we go back to our starting point, the F-string transmission, we realize that we could just as easily have taken the strings to be diagonal in target space with winding $m(1)+n(2)$ along the $x_{1}, x_{2}$-cycles. It would be very easy to repeat the calculation of section 2 in this case. The $\mathrm{SL}(2, \mathrm{Z})$ transformation (34) relates this to a diagonal version of (36). The power of introducing diagonal winding is that by compactifying $x_{4}$ and by using a sequence of dualities $T_{3} T_{4} S T_{1}$, we can end up with a quite complicated process

$$
\begin{aligned}
& p_{1} m(1234) D 4+q_{1} m(12) D 2+p_{1} n(34) D 2+q_{1} n D 0 \\
& p m(\stackrel{1234)}{\longleftarrow}+\underset{+q m(12) D 2+p n(34) D 2+q n D 0}{\longrightarrow} \longrightarrow \longrightarrow
\end{aligned}
$$

involving $\mathrm{D}(4+2+0)$-transfer between $\mathrm{D}(4+2+0)$-branes. Note that in particular it involves M-momentum transfer between D4-branes? It is not obvious how to accomplish this by approaching the problem as one of finding a suitable non-perturbative configuration in 4+1-dimensional field theory.

\footnotetext{
${ }^{8}$ In this particular case M-momentum transfer always comes along with some D2-brane transfer due to the manner in which lower dimensional branes bind to D4-branes.
} 
So, we conclude that a variety of non-perturbative transfer processes can be analyzed quantitatively by exploiting dualities to relate them to the simpler perturbative process of electric flux transfer between D-branes. In particular, one can study M-momentum transfer between gravitons (37), membranes (39) and longitudinal fivebranes (41) via this route.

\section{Acknowledgements}

We would like to thank M. Aganagic, S. Cherkis, E. Gimon, and T. Kärki for discussions. We are grateful for the hospitality of the Institute for Theoretical Physics in Santa Barbara, where a part of this work was carried out. This research was supported in part by the National Science Foundation under Grant No. PHY94-07194.

\section{References}

[1] T. Banks, W. Fischler, S.H. Shenker and L. Susskind, M theory as a matrix model: a conjecture, Phys. Rev. D55, 5112 (1997), (hep-th/9610043).

[2] P. Pouliot and J. Polchinski, Membrane scattering with M-momentum transfer, Phys. Rev. D56, 6601 (1997), (hep-th/9704029).

[3] N. Dorey, V. Khoze, and M. Mattis, Multi-Instantons, three-dimensional gauge theory, and the Gauss-Bonnet-Chern theorem, Nucl. Phys. B502, 94 (1997), (hep-th/9704197). Note that discrepancies arise upon generalizing the calculation to curved space: J. de Boer, K. Hori, and H. Ooguri, Membrane scattering in curved space with $M$ momentum transfer, hepth/9802005).

[4] E. Keski-Vakkuri and P. Kraus, Notes on branes in Matrix theory, Nucl. Phys. B510, 199 (1998), (hep-th/9706196).

[5] T. Banks, W. Fischler, N. Seiberg and L. Susskind, Instantons, scale invariance and Lorentz invariance in Matrix theory, Phys. Lett. B408, 111 (1997), (hep-th/9705190).

[6] O.J. Ganor, S. Ramgoolam and W. Taylor IV, Branes, fluxes and duality in M(atrix)-Theory, Nucl. Phys. B492, 191 (1997), (hep-th/9611202). 
[7] G. Lifschytz, A note on the transverse five-brane in M(atrix) theory, Phys. Lett. B409 124 (1997). (hep-th/9703201).

[8] J. Schwinger, Phys. Rev. 82, 664 (1951); 93, 615 (1954).

[9] M.J. Duff and K.S. Stelle, Multimembrane solutions of D=11 supergravity, Phys. Lett. B253, 113 (1991).

[10] C. Bachas and M. Porrati, Pair creation of open strings in an electric field Phys. Lett. B296, 77 (1992), (hep-th/9209032); C. Bachas, (Half) a lecture on D-branes, hep-th/9701019.

[11] M.R. Douglas, D. Kabat, P. Pouliot and S.H. Shenker, D-branes and short distances in string theory, Nucl. Phys. B485, 85 (1997), (hep-th/9608024).

[12] I. Chepelev and A.A. Tseytlin, Long-distance interactions of D-brane bound states and longitudinal 5-brane in M(atrix) theory, Phys. Rev. D56, 3672 (1997), (hep-th/9704127).

[13] D. Kabat and W. Taylor IV, Spherical membranes in Matrix theory, hepth/9711078.

[14] W. Taylor IV, D-brane field theory in compact spaces, Phys. Lett. B394, 283 (1997), (hep-th/9611042).

[15] C.R. Stephens, The Hawking effect in abelian gauge theories, Ann. Phys. 193, 255 (1989).

[16] N.D. Birrell and P.C.W. Davies, Quantum fields in curved space, Cambridge University Press (1982).

[17] D. Berenstein and R. Corrado, M(atrix)-Theory in various dimensions, Phys. Lett. B406, 37 (1997), (hep-th/9702108).

[18] J.H. Schwarz, An SL(2,Z) multiplet of type IIB superstrings, Phys. Lett. B360, 13 (1995), Erratum ibid B364, 252 (1995), (hep-th/9508143); E. Witten, Bound states of strings and p-branes, Nucl. Phys. B460, 335 (1996), (hep-th/9510135). 
[19] W. Taylor IV, Lectures on D-branes, gauge theory and M(atrices), hepth/9801182. 